\title{
Explorations and 3D models of Atmospheric and Suspension Plasma Spraying coatings microstructure
}

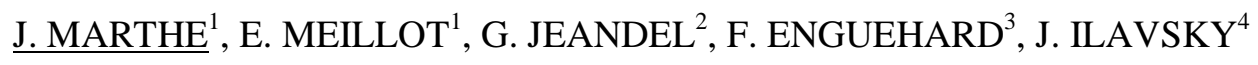

(1) CEA/DAM, Le Ripault, F-37260 Monts, France

(2) LEMTA, Av. de la forêt de Haye, BP 160, F-54504 Vandœuvre-lès-Nancy, France

(3) Ecole Centrale Paris, Laboratoire EM2C, UPR 288 ECP-CNRS, Grande Voie des Vignes, F-92295

Chatenay-Malabry, France

(4) Argonne National Laboratory, 9700S. Cass Avenue, bldg 434D, Argonne, IL 60439, USA

\begin{abstract}
Plasma-spraying processing provides material with typical and complex microstructure. For simulating the mechanical, electrical, optical... properties of such materials, it is necessary to determine the numerical representation of the microstructure. Some techniques, like microtomography, give directly an image of the porosity. Although such methods are convenient, they don't allow modifications of the obtained structure, especially in order to observe the influence of the porosity parameters on the material properties. This work investigates the microstructure of Atmospheric Plasma Spraying (APS) and Suspension Plasma Spraying (SPS) coatings thanks to several analysis techniques such as Scanning Electron Microscopy, image analysis, $\mathrm{Hg}$ porosimetry or Ultra Small Angle X-ray Scattering (USAXS). From the different obtained result, a flexible $3 \mathrm{D}$ representation of each coating is computed.
\end{abstract}

\section{Introduction}

Resulting of spreading and stacking of fused particle onto a substrate, the plasma-sprayed materials have typically heterogeneous, usually porous, microstructure. The non-perfect stacking generates globular pores and interlamellar cracks. Moreover, the quick cooling of the fused particle by the cold substrate implies intralamellar cracks relaxing the residual stresses [1]. This porosity is the cause of the decrease or the modification of coatings behavior, such as mechanical 
or electrical properties. In order to improve these performances, simulations are performed to understand the link between the microstructure and the required properties. The first difficulty for the prediction of plasma sprayed coatings properties is to describe their microstructures. In fact, the relevance of the simulation is linked with the refinement for describing the material. Previous works are based on reconstruction from statistical characterization or on the simulation of the generation of the coating. For 2D representations, the globular pores can be represented by spherical inclusion in homogenous matrix [2] and the cracks by elliptic shapes [3]. SEVIASTIANOV and al. models the porosity by a statistics distribution of pores and oriented cracks [4].

The evolution of the computing tools allows generating tridimensional representation by the spreading of alumina lamella and the creation of defects in the structure [5]. From this microstructure, the author determines the dielectric permittivity of alumina plasma-sprayed coatings. The micro-tomography analysis explores the microstructure of a material by successive removal of matter. AMSELLEM and al. [6] used this technique to predict the mechanical and electrical properties of alumina plasma sprayed coatings. Although this method is very interesting, the actual resolution doesn't allow the observation of cracks and the nanostructured organization. Moreover, the result of micro tomography is fixed and it is impossible to observe the influence of the microstructure characteristics on the modeled properties.

One of the most versatile and recent models is obtained from pictures analysis and 3D reconstruction. The used shapes are spheres for globular pores and parallelepiped with low thickness for the cracks [7].

\section{1) General principle of the microstructure generation}

The field is meshed in a Cartesian coordinate system (x, y, z) as thinner as possible (step $\mathrm{S}$ ) according to the computer memory and each point $\mathrm{M}$ of the meshing is located with its 
coordinates $\left(X_{M} ; Y_{M} ; Z_{M}\right)$. The size of the cubic box for describing the material is delimited by origin $\mathrm{O}$ and $M_{\lim \left(X_{\lim }^{3} Y_{\lim }^{3} Z_{\text {lim }}\right)}$ (Figure 1). The meshing is described by a 2-dimensions matrix $\mathrm{P}$ with $\mathrm{X}$ rows and $\mathrm{X}^{*} \mathrm{Y}$ lines, where :

$X=\frac{X_{\text {lim }}}{S} \quad Y=\frac{Y_{\text {lim }}}{S} \quad Z=\frac{Z_{\text {lim }}}{S}$

For each point $\mathrm{M}$, the criterion is 1 when there is matter and 0 if the point is inside a pore.

The most complicated 3D closed shape with a relative simple equation is ellipsoid. By modifying the shape factor $f$ of the ellipsoid, it is possible to approximate globular pores $(f$ close to 1$)$ and cracks ( $f$ close to 0 ) in plasma-sprayed coating.

In a Cartesian coordinate system $(\mathrm{X}, \mathrm{Y}, \mathrm{Z})$, the standard equation of an ellipsoid centered at the point $\Omega\left(X_{\Omega} ; Y_{\Omega} ; Z_{\Omega}\right)$ and with semi-principal axis a, b and c is:

$$
\frac{\left(X-X_{\Omega}\right)^{2}}{a^{2}}+\frac{\left(Y-Y_{\Omega}\right)^{2}}{b^{2}}+\frac{\left(Z-Z_{\Omega}\right)^{2}}{c^{2}}=1
$$

The point $M\left(X_{M} ; Y_{M} ; Z_{M}\right)$ is inside the ellipsoid if:

$$
\frac{\left(X_{M}-X_{\Omega}\right)^{2}}{a^{2}}+\frac{\left(Y_{M}-Y_{\Omega}\right)^{2}}{b^{2}}+\frac{\left(Z_{M}-Z_{\Omega}\right)^{2}}{c^{2}}<1
$$

In this case, $\mathrm{P} \mathbb{}\left(Y_{M} \times Y_{M} ; X_{M}\right)=\mathbf{0}$, otherwise $\mathrm{P} \mathbb{Z}\left(Y_{M} \times Y_{M} ; X_{M}\right)=\mathbf{1}$.

\section{2) Description of microstructure coatings}

\section{a) Bilayer alumina for optical applications}

In order to illustrate this work with a real example, the microstructure of coating, developed in previous work for optical applications [8], has been investigated. Samples are constituted by a double-layer coating, deposited onto a substrate. The first layer (called APS layer) is sprayed by atmospheric plasma spraying from $\alpha$-alumina powder (micrometric size range $-22+5 \mu \mathrm{m}$ 
H.C.Starck, Germany). The top layer (called SPS layer) is manufactured by Suspension Plasma Spraying from $\gamma$-alumina with nanometric size centered on $100 \mathrm{~nm}$. The two layers are manufactured with the same F4-VB plasma torch (Sulzer-Metco AG, Wohlen, Switzerland) equipped with $6 \mathrm{~mm}$ internal diameter nozzle. At the end of the process, the coatings are submerged in liquid nitrogen in order to create a thermal shock to separate the coatings from the substrates.

The elaboration process parameters and the characterizations of the plasma-sprayed bi-layer coating are explained in detail in [8] and [9].

This example shows the treatment of multiscale microstructure: the APS layer is microstructured while the SPS layer is nanostructured. These two layers have to be characterized separately with different tools.

\section{b) SEM observations and images treatments}

The coating microstructures are observed by optical microscopy by Scanning Electron Microscope (SEM) (XL30, Koninklije Philips Electronics N.V., Amsterdam, The Netherlands) on polished and fractured cross sections.

The SEM observations are one of the only ways to observe directly the shape of the different heterogeneities. The Figure 2 shows a typical lamellar and tangled structure for the APS coating. Spherical particles, resulting of partially-melted powder can be also observed. The thickness of the lamella can be estimated around 2 microns and the length of intralamellar cracks is around 15 microns. For the SPS layer, the resolution of the SEM is not enough to observe heterogeneities inside the coating. The microstructure appears as a compact collection of globular particles and the porosity is invisible. 
In order to collect more quantitative and statistic information on the microstructure of APS coating, SEM polished cross sections micrographs treatments are performed for APS layer from 10 pictures. A threshold of the picture is realized to obtain bi-chromatic pictures (Figure 4). The software Image $\mathbf{J}$ allows to detect and to class the different shapes in each picture according the shape factor ( $f=\mathbf{1}$ for a disk and $f \rightarrow \mathbf{0}$ for a crack). Moreover, the software calculates the Feret diameter of each shape. The Feret diameter corresponds to the distance between the two parallel planes restricting the object perpendicular to that direction and this value estimates the diameter of globular pores or the length of the cracks.

The heterogeneities are divided in three classes (Figure 5):

- The heterogeneities with $f \in[0 ; 0.2]$ are associated to the cracks. They are majoritary in number $(60 \%)$. The analysis of the size shows a distribution of the Feret diameter, centered on 20 microns. This average length is coherent with the estimated value by SEM observation (around 15 microns). The analysis detects also longer heterogeneities (up to 50 microns) due to the interconnectivity of the porosity.

- The globular pores with $f \in[0.8 ; 1]$ represent a low quantity of the detected heterogeneities (only 3\%). They have a diameter between 1 and 5 microns.

- The rest of the porosity, with $f \in[0.2 ; 0.8]$, have an intermediary shapes and represents $37 \%$ of the heterogeneities. Their sizes are centered on 10 microns.

This analysis estimates the relative proportion between cracks and globular pores (60/40). However, this method is approximate because the result is directly link to the used power-field. In addition, the resolution is not enough to apply this technique for nanostructured material such as SPS coatings. These limitations imply the use of multi-scale characterization tools in order to describe precisely the porosity of plasma-sprayed coatings. 


\section{c) Hg Porosimetry}

The determination of pores size distribution by Mercury Intrusion porosimetry is performed on Micromeritics Autopore III 9410. The measurable pore sizes range between a few nanometers and up to $100 \mu \mathrm{m}$ by applying pressure from 0.04 up to $400 \mathrm{MPa}$ and assume mercury wetting angle of $130^{\circ}$. The pores size distributions of the alone layers and of the bi-layer system size are shown in Figure 6. For the APS layer, a large band of pore sizes spreading from 80 to $400 \mathrm{~nm}$ and, centred at $200 \mathrm{~nm}$, is observed. This distribution shows also the presence of large pores with size between $500 \mathrm{~nm}$ to $3 \mu \mathrm{m}$. The distribution of pores in SPS layer is obtained by subtraction of bi-layer and APS coatings curves. In fact, the SPS coating is too thin and too fragile to resist alone against the $\mathrm{Hg}$ pressure. A large quantity of small pores is observed in the range size below $100 \mathrm{~nm}$ with two bands (at 20 and $40 \mathrm{~nm}$ ).

\section{d) USAXS for SPS layer}

In order to determine pore size distributions in SPS layer, Ultra-Small Angle X-ray Scattering (USAXS) analysis are performed [10]. The experiment was conducted at the ChemMatCARS beamline 15-ID at the Advanced Photon Source, Argonne National Laboratory. The instrument uses X-ray energy of $16.9 \mathrm{keV}$ to optimize the sample transmission and minimize effects of multiple scattering. Beam size is about $2 \mathrm{~mm}$ (width) x 0.8 (height). Sample thickness is about $0.1 \mathrm{~mm}$, giving total scattering volume of sample characterized around $0.16 \mathrm{~mm}^{3}$ The pores can be modeled thanks to the Irena data analysis package [11] by different shapes. The Figure 7 shows the obtained results according to the shape factor $f$. For relatively spherical shape ( $\mathrm{f}=0.6$, $0.8,1)$, the distributions are very close and centered between 6 and $8 \mathrm{~nm}$. When the shape factor $f$ decreases, the average size increases to conserve a constant volume. There is about one size order between $f=\mathbf{1}$ and $f=0.1$. 
This results show the importance of the shape with which the porosity is modeled. Moreover, there are in plasma spared coatings different shapes of heterogeneities and the pores are modeled with only one shape.

\section{3) 3D representations}

From these results, it is possible to propose 3D representations for APS and SPS coatings. For APS coating, globular pores are represented by ellipsoid with a factor shape $f \in[0.8 ; 1]$ and the sizes of the 3 axis a, b, and c are between 1 and 5 microns. The cracks are modeled by ellipsoid with $f \in] 0 ; 0.1]$ and a thickness of 0.25 microns. The length of horizontal interlamellar cracks is 20 microns and the length of vertical cracks is equal to the thickness of a lamella: 3 microns. The ratio between globular pores and cracks is $40 / 60$ and the global porosity is $13 \%$. The centers of ellipsoid are set randomly in space and the contacts between the different heterogeneities are implicitly integrated because the porosity is interconnected (Figure 8). In order to represent the microstructure of SPS layer, the assumption of using the USAXS results with spherical pores has been chosen. The model respects this USAXS distribution (Figure 9a) and the global porosity of 15\% (Figure 9b). An experimental design will define the most appropriate shape in order to obtain a concordance between simulated and experimental properties.

\section{Conclusions}

This work presents a versatile model for 3D representation of plasma-sprayed coatings. Thanks to SEM observations, images analysis, $\mathrm{Hg}$ Intrusion porosimetry, it is possible to quantify the different parameter of the APS coating porosimetry. Although, the resolution of current tools (SEM, micro-tomography) is not enough to observe the nano-heterogeneites inside the SPS coating, USAXS analysis allow to have an idea of the pore size distribution. 
The global porosity, the nature of heterogeneities, their shapes, their sizes, their relative positions, or their orientations can be selected easily. This model can be implemented in different algorithm in order to predict mechanical, electrical, optical...properties and to identify which parameters of the microstructure are influent.

\section{Acknowledgments}

ChemMatCARS Sector 15 is principally supported by the National Science Foundation/Department of Energy under grant number NSF/CHE-0822838. Use of the Advanced Photon Source is supported by the U.S. Department of Energy, Office of Science, Office of Basic Energy Sciences, under Contract No DE-AC02-06CH11357.

\section{$\underline{\text { References }}$}

[1] P. Fauchais , «Understanding Plasma Spraying », Journal of Physics D: Applied Physics, 37, 2004, pp R86-R108

[2] V. Debout, "Contribution à l'Etude des Propriétés Optiques de Dépôts Plasma de Zircone Yttriée : Influences de leurs Caractéristiques Microstructurales et Physico-chimiques», Thèse de doctorat de l'Université de Limoges, 2007

[3] L. Robin-Carillon, «Etude expérimentale et théorique de l'émission infrarouge de céramique à haute température: Application aux barrières thermiques», thèse de doctorat de 1'Institut National des Sciences Appliquées de Lyon, 2007

[4] I. Sevostianov, M. Kachanov, J. Ruud, P. Lorraine, M. Dubois, «Quantitative characterization of microstructures of plasma-sprayed coatings and their conductive and elastic properties», Materials Science and Engineering A, 386, 2004, pp 164-174

[5] S. Beauvais, «Etude de l'influence de la porosité sur les propriétés électriques de dépôts réalisés par projection plasma», Thèse de doctorat de l'Ecole Nationale Supérieure des Mines de Paris, 2003

[6] O. Amsellem, «Simulations $2 d$ et $3 d$ de microstructure d'alumine projetée plasma pour l'étude de ses propriétés mécaniques et électriques», Thèse de Doctorat de l'école des Mines de Paris, 2008 
[7] J. H. Qiao, R. Bolot, H. Liao, P. Bertrand, C. Coddet, «A 3D finite-difference model for the effective thermal conductivity of thermal barrier coatings produced by plasma spraying», International Journal of Thermal Sciences, 65, 2013, pp 120-126

[8] J. Marthe, E. Meillot, G. Jeandel, F. Enguehard and J. Ilavsky, "Enhancement of scattering and reflectance properties of plasma-sprayed alumina coatings by controlling the porosity", Surface and Coating Technology, Vol 220, 2013, pp 80-84

[9] J. Marthe, E. Meillot, F. Enguehard and G. Jeandel, "A bi-layer plasma sprayed alumina for optical application", Proceedings from International Thermal Spray Conference and Exposition, 2012, pp 703-707

[10] J. Ilavsky, P. R. Jemian, A. J. Allen, F. Zhang, L. E. Levine, and Gabrielle G. Long, Ultra-smallangle X-ray scattering at the advanced Photon Source, J. Appl. Crystallogr. 42 (3), 2009, p469-479

[11] J. Ilavsky, P. R. Jemian, Irena: tool suite for modelling and analysis of small-angle scattering, J. Appl. Crystallogr., 42 (2), 2009 


\section{List of figures}

Figure 1 : Building of the matrix $\mathrm{P}$

Figure 2: Optical (a) and SEM (b) micrographies of the alumina bilayer coating Figure 3 : SEM micrographies of the APS coating (a) polished cross section and (b) (c) (d) fracture observations

Figure 4 : Threshold of SEM migrographies of APS coating

Figure 5 : Results in number of image analysis on APS coating (a) Global repartition accordiang the shape factor (b) (c) and(d) Repartition of the number of heterogeneities according the Feret diameter and the shape factor

Figure 6: Pores size distributions of APS layer, SPS layer and bi-layer coating measured by Mercury Intrusion Porosimetry

Figure 7 : USAXS Analysis of SPS coating (a) Scattered intensity of X-ray according the scattered vector (b) Pores size distribution (number)

Figure $8: 3 \mathrm{D}$ representation of APS coating (a) global view, (b) side view

Figure 9 : 3D representations of SPS coating (a) Visualisation of USAXS distribution (b) global porosity: $15 \%$ 

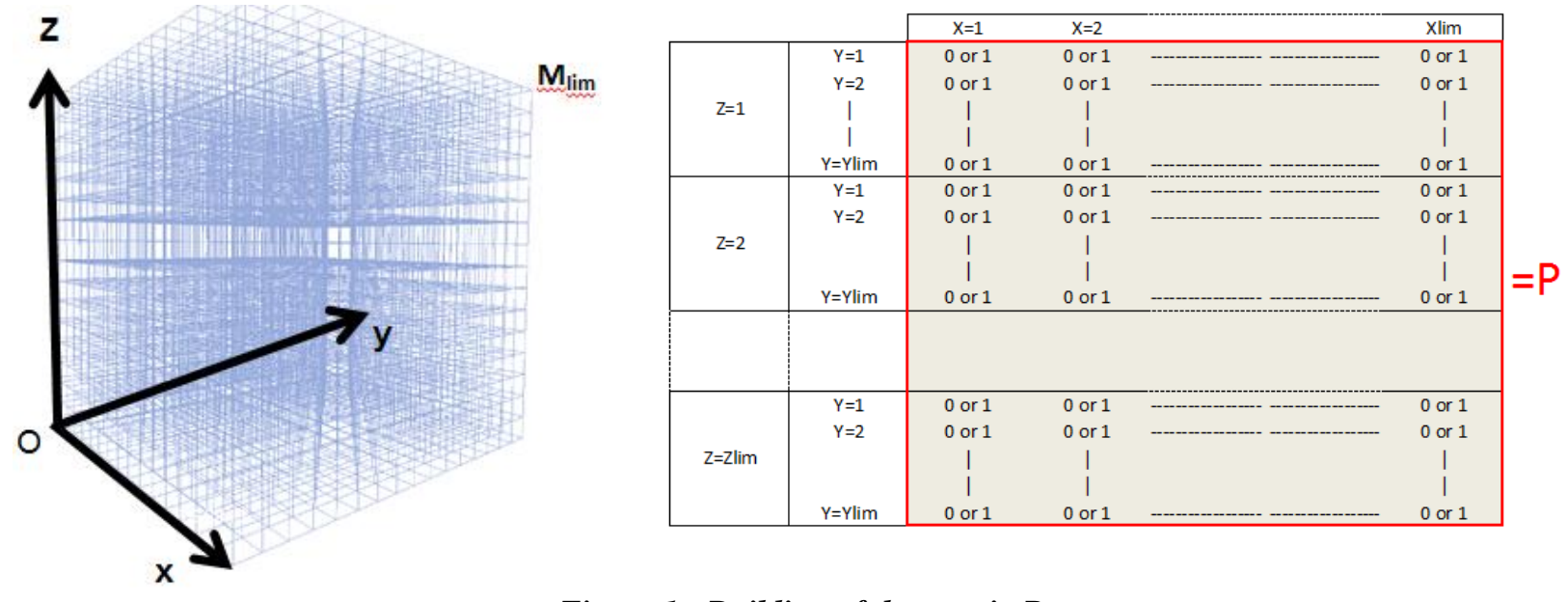

Figure 1 : Building of the matrix $P$ 
(a)

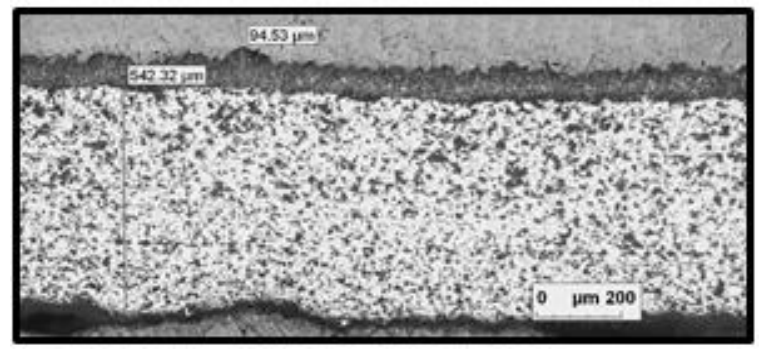

(b)

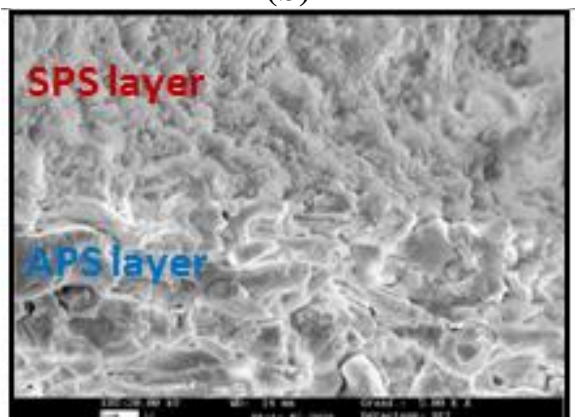

Figure 2: Optical (a) and SEM (b) micrographs of the alumina bilayer coating 
(a)

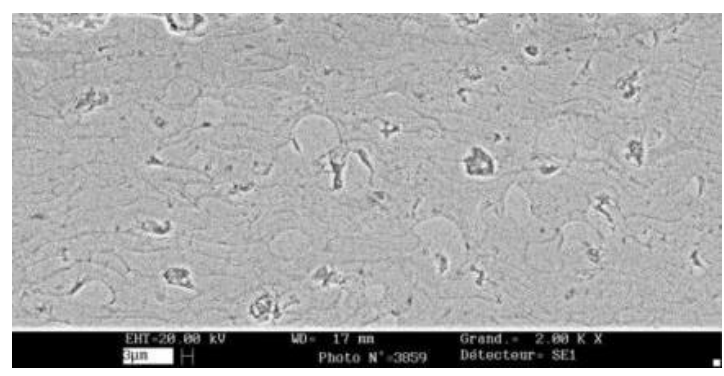

(c)

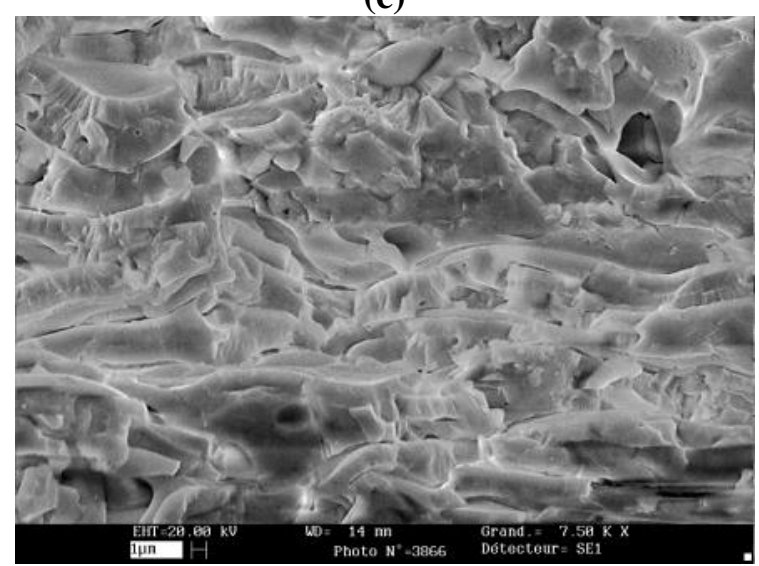

(b)

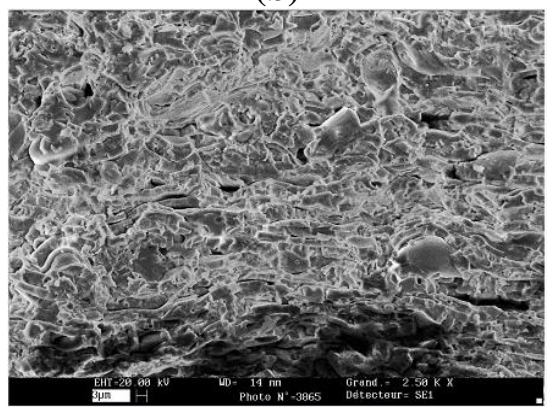

(d)

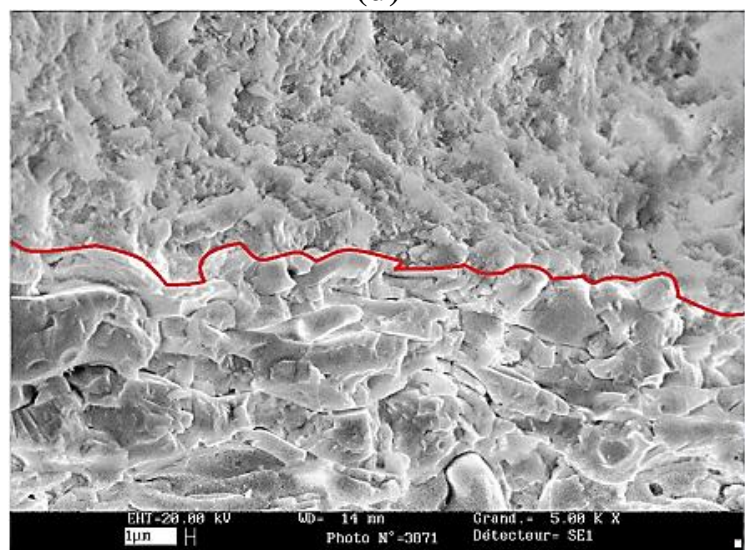

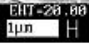

Figure 3 : SEM micrographs of the APS coating (a) polished cross section and $(b)(c)(d)$ fracture observations 

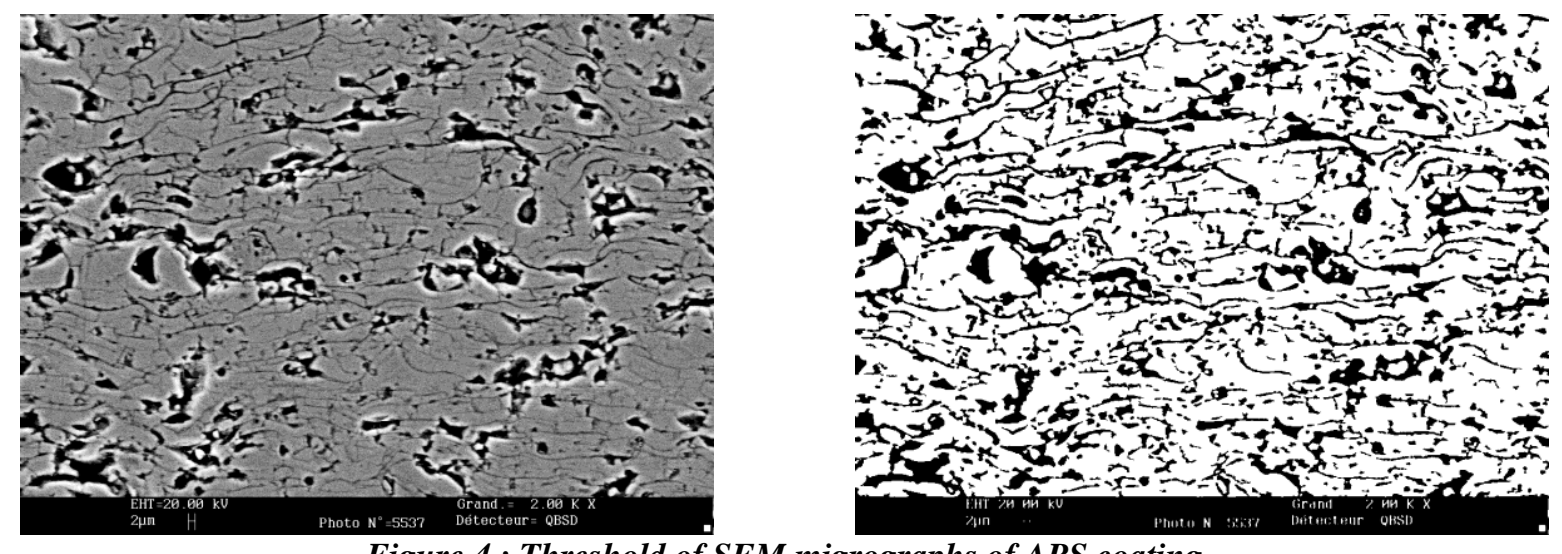

Figure 4: Threshold of SEM migrographs of APS coating 
(a)

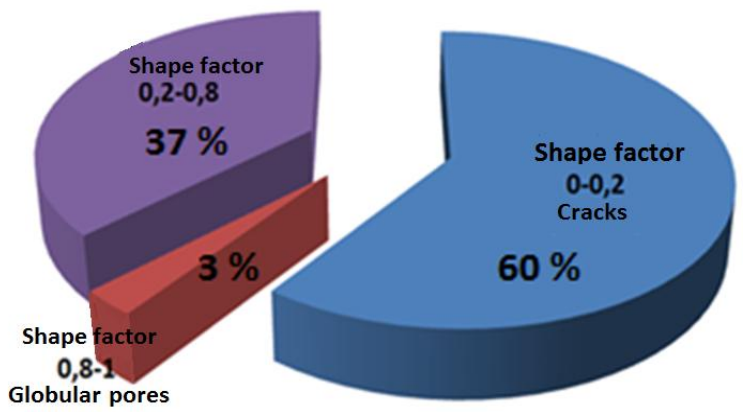

(c)

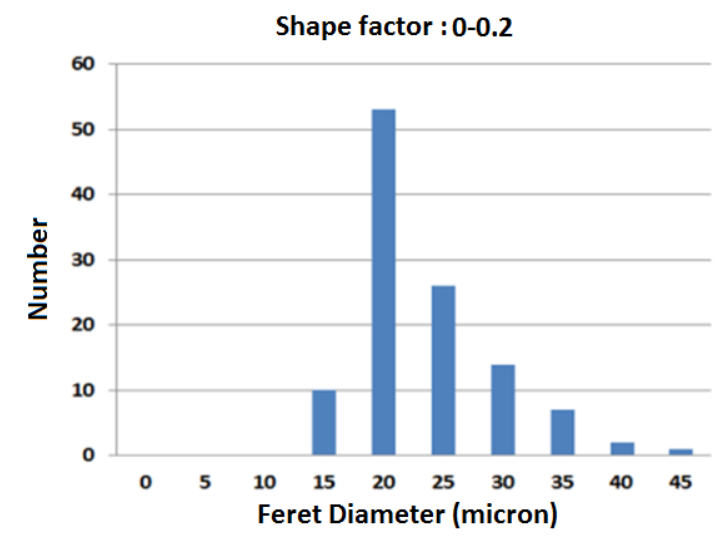

(b)

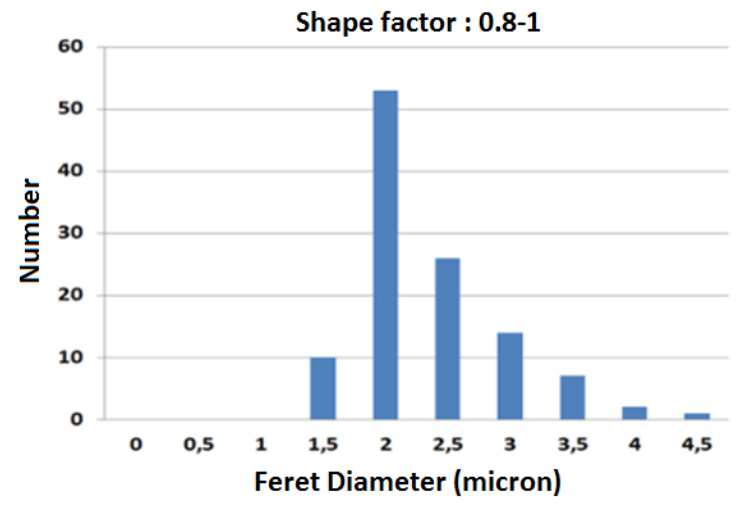

(d)

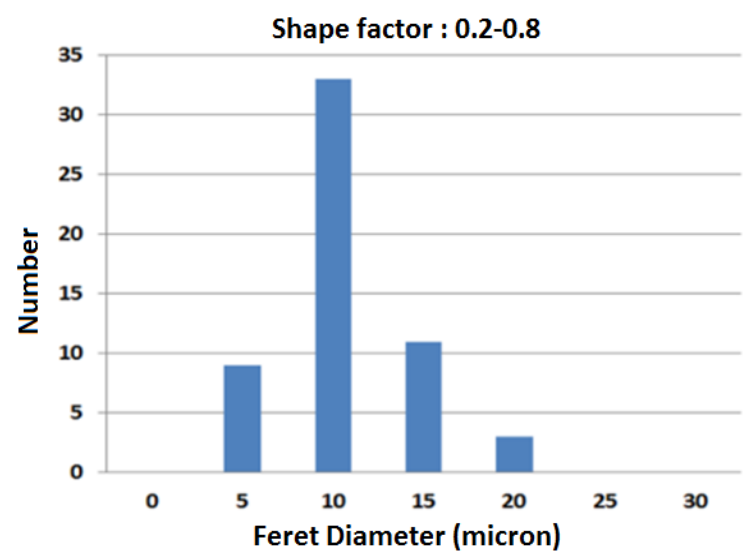

Figure 5 : Results in number of image analysis on APS coating (a) Global repartition according the shape factor (b) (c) and (d) Repartition of the number of heterogeneities according the Feret diameter and the shape factor 


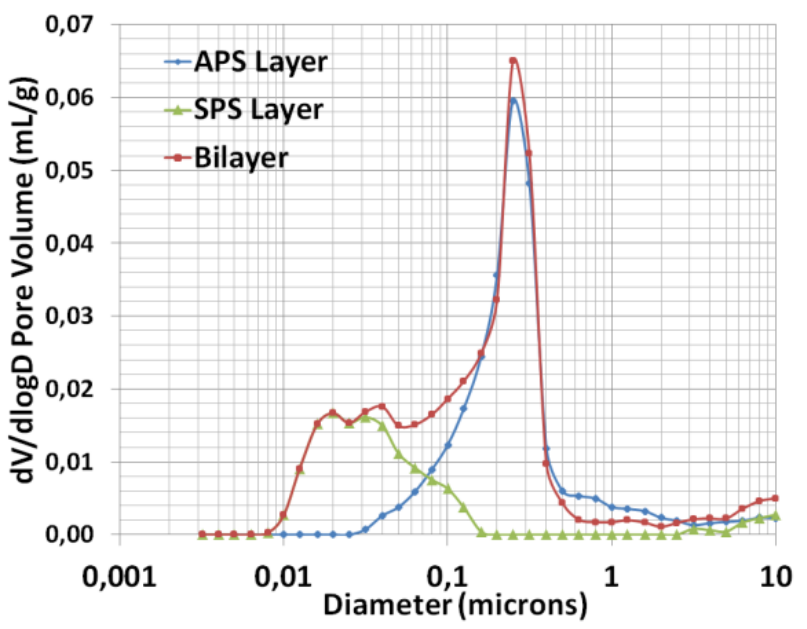

Figure 6: Pores size distributions of APS layer, SPS layer and bi-layer coating measured by Mercury Intrusion Porosimetry 

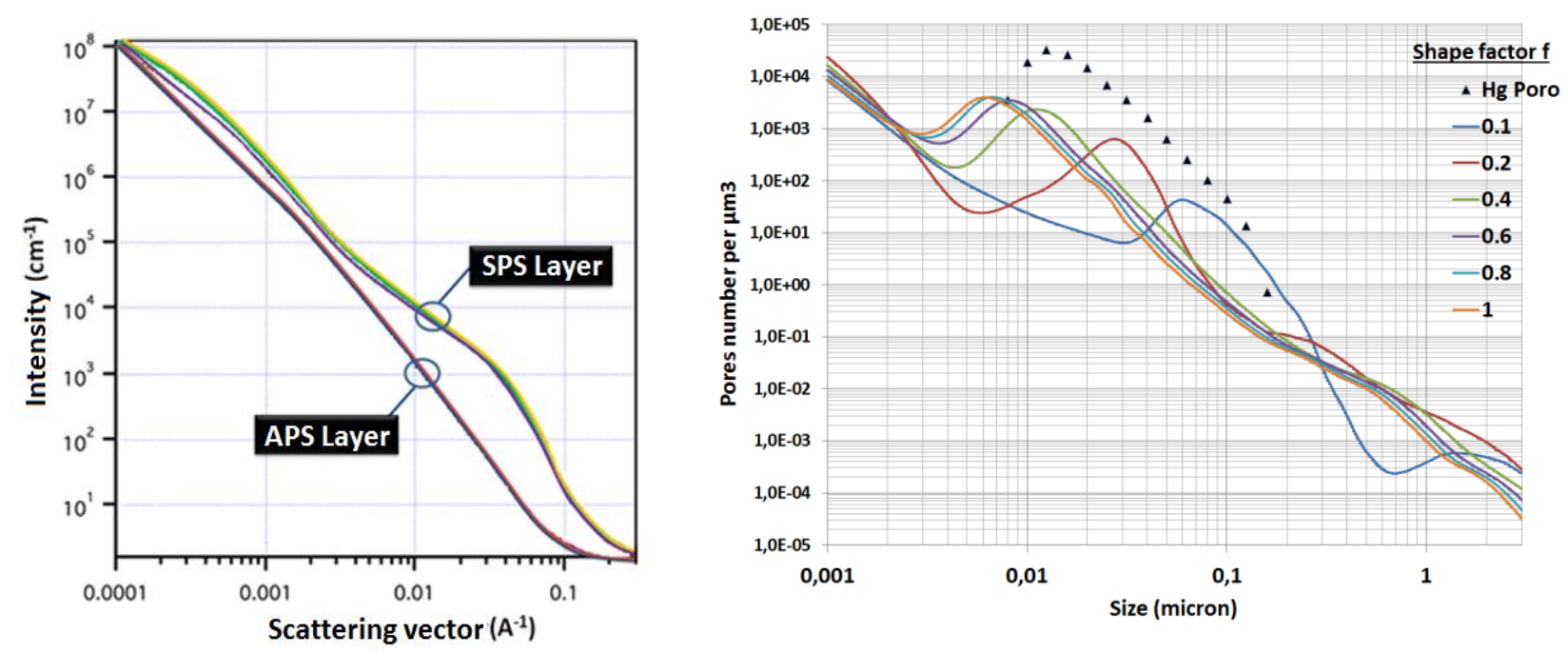

Figure 7 : USAXS Analysis of SPS coating (a) Scattered intensity of X-ray according the scattered vector (b) Pores size distribution (number) 
(a)

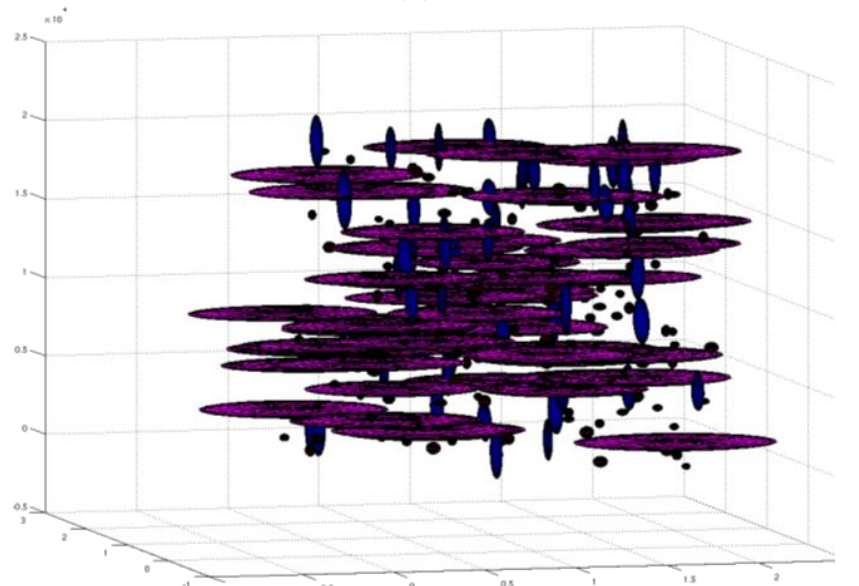

(b)

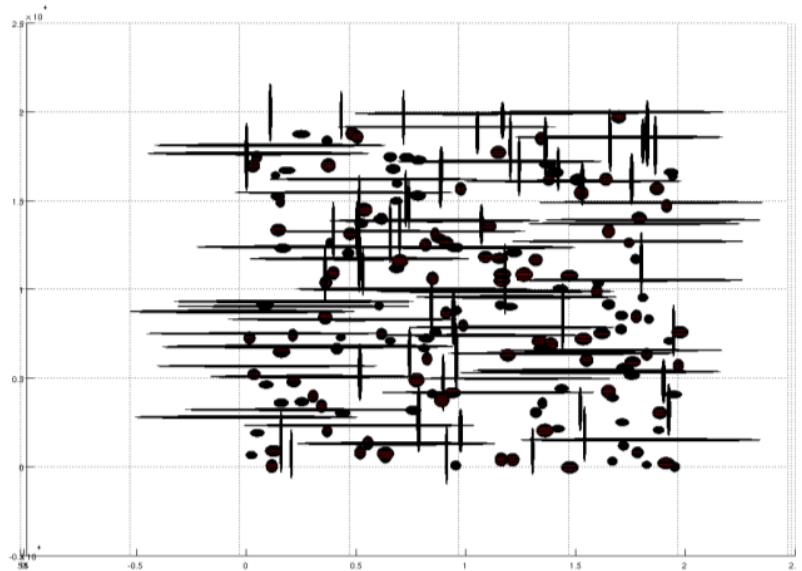

Figure 8:3D representations of APS coating (a) global view, (b) side view 
(a)

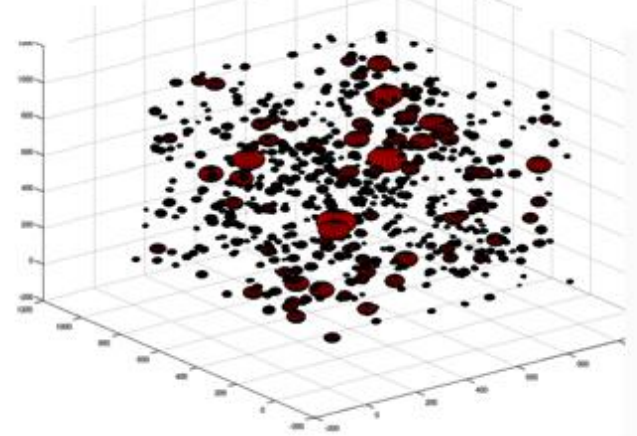

(b)

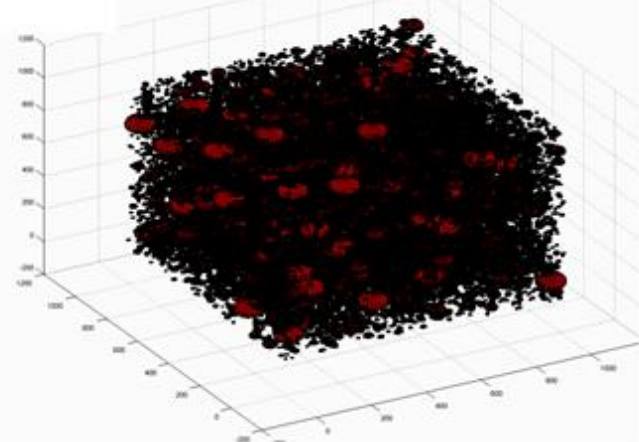

Figure 9:3D representations of SPS coating (a) Visualisation of USAXS distribution (b) global porosity: $15 \%$ 